\title{
Canonical and Grand Canonical Gibbs States for Continuum Systems
}

\author{
Hans-Otto Georgii \\ Institut für Angewandte Mathematik der Universität, \\ D-6900 Heidelberg, Federal Republic of Germany
}

\begin{abstract}
It is shown that for a large class of interactions any canonical Gibbs state satisfying a natural temperedness condition is a mixture of Gibbs states with appropriate activities, and vice versa. Some general results on Gibbs states and canonical Gibbs states are established. In particular, a differential characterization of Gibbs states is given.
\end{abstract}

\section{Introduction}

A state of a many particle system is called a canonical or a (grand canonical) Gibbs state ${ }^{1}$ if its conditional probabilities in bounded volumes are given by the canonical or grand canonical Gibbs distributions, respectively. While the Gibbs states are easier to deal with, the natural candidates for the invariant states under the motion of interacting particles are the canonical Gibbs states. This intuition has been confirmed not only for lattice systems (see [15] and the references in [6]) but also for continuum systems - the relation between canonical Gibbs states and the so-called classical KMS condition recently established by Aizenmann, Goldstein, and Lebowitz ${ }^{2}$ (private communication) is a result in this spirit.

The first question concerning canonical Gibbs states is whether they are mixtures of Gibbs states. For lattice systems an affirmative answer has been given by Thompson, Logan, Shiga, and the author (see $[6,7,15]$ and the references there), and for continuum systems of independent particles by Nguyen and Zessin [11]. In this paper we do the same for continuum systems of interacting particles. The essential ideas are those of [7], but the technical details are rather different.

Now we describe the main result for the special case of shift invariant interactions.

(0.1) Theorem. Suppose that the interaction is given by a translationally invariant finite range potential of one of the following four types:

(PP) Positive pair potential.

\footnotetext{
1 Gibbs states are often called equilibrium states satisfying the DLR-equations.

2 Their interest in canonical Gibbs states for continuum systems stimulated the work presented here.
} 
(DP) Pair potential bounded from below and diverging at the origin faster than $|x|^{-d}$, where $d$ is the dimension.

(CP) Hard core pair potential bounded from below.

(WR) The Widom-Rowlinson potential [16].

Suppose further that $\mu$ is a canonical Gibbs state whose particle density is almost surely finite or, in case (CP), strictly smaller than the density of the densiest packing of the hard balls. Then $\mu$ is a mixture of Gibbs states with respect to this interaction and certain activities. The distribution of the activities is given by the distribution under $\mu$ of a certain function $z($.$) on the configuration space. Further-$ more, under $\mu$ the $\sigma$-fields of the tail events and of the symmetric events almost surely coincide.

The complete statement of the main theorem and related results are collected in Section 5. The key result - the existence of the tail measurable activity indicating function $z($.$) - is proved in Sections 6$ and 7. Section 1 contains the set-up, notations, and a description of the interactions. In Sections 2 and 4 some fundamental results on Gibbs states and canonical Gibbs states are established. In Section 3 we show that Gibbs states exhibit a characteristic behaviour if a particle is fixed at a given site.

\section{Preliminaries}

1.1. The Particle Space. We denote by $S$ the $d$-dimensional Euclidean space $\mathbb{R}^{d}$, $d \geqq 1$, and by $\mathscr{S}$ the $\sigma$-field of Borel sets in $S$. Furthermore, let $\mathscr{L}$ be the set of all bounded $\Lambda \in \mathscr{S}$, and

$$
\overline{\mathscr{L}}=\{\Delta(k): k=1,2, \ldots\}
$$

where

$$
\Delta(k)=\left\{x=\left(x_{1}, \ldots, x_{d}\right) \in S:\left|x_{i}\right| \leqq k(1 \leqq i \leqq d)\right\} .
$$

If $\Lambda$ runs through a certain increasing cofinal subsequence of $\mathscr{L}$, we write $\Lambda \uparrow S$, and if $\Delta$ runs through the sequence $\overline{\mathscr{L}}$, we write $\Delta \uparrow S$.

We fix a certain Radon measure $\sigma$ on $(S, \mathscr{S})$ being atomless in the sense that $\sigma(\{x\})=0$ for all $x \in S$. The usual choice is $\sigma=\lambda$ where $\lambda$ denotes the Lebesgue measure, at least in all cases where translation invariance is needed. Another standard choice is $\sigma=e^{\phi} \lambda$, where the measurable function $x \rightarrow \phi(x)$ has the interpretation of a chemical potential. If we choose $\sigma\left(S \backslash S_{0}\right)=0$ for some $S_{0} \in \mathscr{S}$ then the actual particle space is $S_{0}$ (for barometric problems, e.g., let $S_{0}$ be the upper halfspace). Sometimes we shall need the following condition on $\sigma$ :

$$
\lim _{k \rightarrow \infty} \sigma(\Delta(k+1)) / \sigma(\Delta(k))=1
$$

which is trivially satisfied if $\sigma=\lambda$. Finally, we denote by $\mathscr{L}_{\sigma}$ the set of all $\Lambda \in \mathscr{L}$ with $\sigma(\Lambda)>0$.

1.2. The Configuration Space. We are interested in configurations $\omega$ of indistinguishable particles in $S$ which are locally finite, i.e., in every $\Lambda \in \mathscr{L}$ there are only finitely many particles. Thus we consider the configuration space

$$
\Omega=\{\omega \subset S: \omega(\Lambda)<\infty \text { for all } \Lambda \in \mathscr{L}\}
$$


where

$$
\omega(\Lambda)=N(\Lambda)(\omega)=\operatorname{card}\{x \in \Lambda: x \in \omega\} \quad(\Lambda \in \mathscr{S}) .
$$

The latter expression defines a so-called counting measure, i.e., an integer-valued Radon measure on $(S, \mathscr{S})$, such that $\omega(\{x\}) \leqq 1$ for all $x \in S$. Conversely, any such counting measure defines a unique $\omega \in \Omega$. Hence we can represent a configuration $\omega$ equivalently in both ways, and notations as $x \in \omega$ or $\int \omega(d x) f(x)$ are both meaningful.

For any $\Lambda \in \mathscr{S}, N \geqq 0$, let

$$
\Omega_{\Lambda}^{0}=\{\omega \in \Omega: \omega(S \backslash \Lambda)=0\}
$$

and

$$
\Omega_{\Lambda, N}=\{\omega \in \Omega: \omega(\Lambda)=N\}, \Omega_{\Lambda, N}^{0}=\Omega_{\Lambda, N} \cap \Omega_{\Lambda}^{0} .
$$

Clearly, $\Omega_{\Lambda}^{0}$ is isomorphic to the set of all locally finite subsets of $\Lambda$, and $\Omega$ is isomorphic to $\Omega_{\Lambda}^{0} \times \Omega_{S \backslash \Lambda}^{0}$. If $\omega \in \Omega$ then $\omega_{\Lambda}=\omega \cap \Lambda \in \Omega_{\Lambda}^{0}$ denotes the restriction of $\omega$ to $A$. If $\zeta, \omega \in \Omega$ then $\zeta \omega=\zeta \cup \omega \in \Omega$ is their union. In particular, if $\zeta=\{x\}$ we write $\omega x$ instead of $\omega\{x\}$. 0 denotes the empty configuration.

1.3. Events in $\Omega$. If $\Lambda \in \mathscr{S}$ let $\mathscr{F}_{\Lambda}$ be the $\sigma$-field in $\Omega$ generated by the cylindric sets $\Omega_{V, k}, k \geqq 0, \Lambda \supset V \in \mathscr{L}$, and $\mathscr{F}=\mathscr{F}_{S}$. Clearly, $\mathscr{F}=\mathscr{F}_{\Lambda} \otimes \mathscr{F}_{S \backslash \Lambda}$. It is well-known that there is a Polish topology on $\Omega$ such that $\mathscr{F}$ is the Borel field with respect to this topology, see [10], e.g. An essential role plays the tail field

$$
\mathscr{F}_{\infty}=\bigcap_{\Lambda \in \mathscr{L}} \mathscr{F}_{S \backslash \Lambda} \cdot
$$

If $\Lambda \in \mathscr{L}$ the $\sigma$-field $\mathscr{G}_{\Lambda}$ of $\Lambda$-symmetric events is the $\sigma$-field generated by the events

$$
\Omega_{\Lambda, N} \cap A \quad\left(N \geqq 0, A \in \mathscr{F}_{S \backslash A}\right),
$$

and the $\sigma$-field of symmetric events is

$$
\mathscr{G}_{\infty}=\bigcap_{\Lambda \in \mathscr{L}} \mathscr{G}_{\Lambda} .
$$

1.4. States on $\Omega$. A probability measure $\mu$ on $(\Omega, \mathscr{F})$ is called a state or a simple point process. We are concerned only with states being locally absolutely continuous with respect to the Poisson point process $\pi$ with intensity measure $\sigma$. We write $\mu \ll v$ if $\mu$ is absolutely continuous w.r. to $v$, and local absolute continuity means that $\mu \ll v$ on $\mathscr{F}_{\Lambda}$ for all $\Lambda \in \mathscr{L}$. The Poisson point process $\pi$ with intensity measure $\sigma$ is defined by

$$
\pi \mid \Omega_{\Lambda, N}^{0}=e^{-\sigma(\Lambda)} \frac{1}{N !} \sigma\left(d x_{1}\right) \ldots \sigma\left(d x_{N}\right) \prod_{1 \leqq i \leqq N} 1_{\Lambda}\left(x_{i}\right)
$$

where $N \geqq 0$ and $\Lambda \in \mathscr{L}$. Note that the right hand side is carried by $\Omega_{\Lambda, N}^{0}$ since $\sigma$ is atomless. An essential property of $\pi$ is that if $\Lambda \in \mathscr{L}$ is the disjoint union of $\Lambda_{1}$ and $\Lambda_{2}$ then $\pi\left|\Omega_{\Lambda}^{0}=\pi\right| \Omega_{\Lambda_{1}}^{0} \otimes \pi \mid \Omega_{\Lambda_{2}}^{0}$, that is, $\mathscr{F}_{\Lambda_{1}}$ and $\mathscr{F}_{\Lambda_{2}}$ are independent under $\pi$.

1.5. The Particle Interaction. An interaction potential is a measurable function $U$ on $\Omega_{f}=\cup\left\{\Omega_{\Lambda}^{0}: \Lambda \in \mathscr{L}\right\}$ such that $U(0)=0$ and

(U1) Stability. There is a constant $B \geqq 0$ such that $U(\omega) \geqq-B \omega(S)$ for all $\omega \in \Omega_{f}$. 
(U2) Finiteness. There is a norm $\|\cdot\|$ on $S$ such that $\{x \in S:\|x\| \leqq 1\}$ is convex, and a constant $r \geqq 0$ such that $U(\omega)<\infty$ if and only if $\omega \in \Omega_{r}$ where $\Omega_{r}=$ $\{\omega \in \Omega:\|x-y\| \geqq r$ if $x, y \in \omega$ and $x \neq y\}$.

Clearly, $\Omega_{0}=\Omega$. If $r>0$ then $U$ is called a hard core potential, and the particles are imagined to have the shape of a $r / 2-\|$.$\| -ball.$

If $r=0$ then we shall need for our main result that $U$ is of finite range. For the sake of simplicity, we shall suppose this property throughout the paper, i.e.,

(U3) Finite Range. There is a constant $R>0$ such that for all $\omega \in \Omega$ and all $\Lambda \in \mathscr{L}$ such that $\omega_{S \backslash \Lambda} \in \Omega_{r} U\left(\omega_{\Delta}\right)-U\left(\omega_{\Delta \backslash \Lambda}\right)=U\left(\omega_{\bar{\Lambda}}\right)-U\left(\omega_{\partial \Lambda}\right)$ whenever $\bar{\Lambda} \subset \Delta \in \mathscr{L}$.

Here $\bar{\Lambda}=\Lambda \cup \partial \Lambda$, and $\partial \Lambda$ is the set of all $x \in S \backslash \Lambda$ whose Euclidean distance from $\Lambda$ is not greater than $R$.

Mostly we are concerned with pair potentials. $U$ is called a pair potential if there is a measurable symmetric function $\Phi$ on $S \times S$ such that for all $\omega \in \Omega_{f}$

$$
U(\omega)=\frac{1}{2} \sum_{x, y \in \omega, x \neq y} \Phi(x, y) .
$$

$\Phi$ is assumed to have the properties

(P1) $\Phi(. .) \geqq$.$-C for some constant C \geqq 0$.

(P2) For all $a>r, \sup _{y \in S} \int_{\|x-y\| \geqq a} \sigma(d x) \Phi_{+}(x, y)<\infty$

where $\Phi_{+}=\max (\Phi, 0)$. Our main result shall be proved for the following four types of potentials.

(PP) Positive Pair Potentials. $\Phi<\infty$, i.e., $r=0$ in (U2), and $C=0$ in (P1), hence $B=0$ in (U1).

(DP) Divergent Pair Potentials. $\Phi<\infty$, i.e., $r=0$ in (U2), and there is a decreasing function $\psi$ on $] 0, R]$ such that $\Phi(x, y) \geqq \psi(|x-y|)$ for all $x \neq y$ and $\psi(r) r^{d} \rightarrow \infty$ if $r \rightarrow 0^{3}$.

(CP) Hard Core Pair Potentials. $r>0$ in (U2), i.e., $\Phi(x, y)=\infty$ if and only if $|x-y|<r$.

(WR) Widom-Rowlinson Type Potentials. There is a measure $\tau$ on $(S, \mathscr{S})$ such that $\tau \leqq c \lambda$ for some $c>0$, and a set $0 \in K \in \mathscr{L}$ such that

$U(\omega)=\tau\left(\bigcup_{x \in \omega}(K+x)\right)-\sum_{x \in \omega} \tau(K+x) \leqq 0$

where $K+x=\{y+x: y \in K\}^{4}$.

It follows easily from (U3) that the potentials (PP), (CP), and (WR) are not only stable but strongly stable in the sense that for all $x \in S$ and $\omega \in \Omega_{r}$

$$
U(x \mid \omega)=U\left(x \omega_{\{\bar{x}\}}\right)-U\left(\omega_{\partial\{x\}}\right) \geqq-B
$$

for some constant $B \geqq 0$.

Sometimes we shall consider translationally invariant interactions. We shall indicate this condition by writing $(\mathrm{PP})_{\theta},(\mathrm{DP})_{\theta},(\mathrm{CP})_{\theta}$, or $(\mathrm{WR})_{\theta}$, respectively. Here $\theta$ denotes the shift group $\left(\theta_{x}\right)_{x \in S}$ acting on $\Omega$ and defined by $\theta_{x} \omega=\{x+y$ : $y \in \omega\}$.

3 This together with (U3) implies (U1), see 3.2.8 in [13].

$4 \quad U$ satisfies (U3) with $R=\operatorname{diam} K$. 


\section{Gibbs States}

In this and the following section we let $\sigma \equiv 0$ be an arbitrary atomless measure on $(S, \mathscr{S})$ and $U$ be an interaction potential satisfying (U1), (U2), (U3).

For any $z>0$ and $\Lambda \in \mathscr{L}$ the Gibbs distribution in $\Lambda$ with activity $z$ and boundary condition $\omega \in \Omega$ is the probability measure on $\left(\Omega, \mathscr{F}_{A}\right)$ defined by its RadonNikodym density with respect to $\pi \mid \mathscr{F}_{\Lambda}$ :

$$
f_{\Lambda}^{z}(\zeta \mid \omega)=\left\{\begin{array}{l}
Z_{\Lambda}(z, \omega)^{-1} z^{\zeta(\Lambda)} \exp \left[-U\left(\zeta_{\Lambda} \omega_{\partial \Lambda}\right)\right] \text { if } \quad \omega_{S \backslash \Lambda} \in \Omega_{r} \\
1 \quad \text { otherwise }
\end{array}\right.
$$

where the grand canonical partition function

$$
\begin{aligned}
e^{\sigma(\Lambda)} Z_{\Lambda}(z, \omega) & =e^{\sigma(\Lambda)} \int \pi(d \zeta) z^{\zeta(\Lambda)} \exp \left[-U\left(\zeta_{\Lambda} \omega_{\partial \Lambda}\right)\right] \\
& =\sum_{N=0}^{\infty} \frac{z^{N}}{N !} \int_{\Lambda^{N}} \sigma\left(d x_{1}\right) \ldots \sigma\left(d x_{N}\right) \exp \left[-U\left(x_{1} \ldots x_{N} \omega_{\partial \Lambda}\right)\right] \geqq e^{-U\left(\omega_{\partial \Lambda}\right)}
\end{aligned}
$$

is finite due to (U1). We include the case $z=0$ by setting

$$
f_{\Lambda}^{0}(\zeta \mid \omega)= \begin{cases}1 & \text { if } \zeta(\Lambda)=0 \\ 0 & \text { otherwise }\end{cases}
$$

The following consistency property is easily checked.

$$
f_{\Delta}^{z}(\zeta \mid \omega)=f_{\Lambda}^{z}\left(\zeta \mid \zeta_{\Delta} \omega_{S \backslash \Delta}\right) \int \pi(d \alpha) f_{\Delta}^{z}\left(\alpha_{\Lambda} \zeta_{S \backslash \Lambda} \mid \omega\right)
$$

whenever $\Lambda \subset \Delta \in \mathscr{L}, \zeta, \omega \in \Omega$.

If $\mu$ is a measure on $(\Omega, \mathscr{F})$, define the measure $\tilde{\mu}$ on $(\Omega \times \Omega, \mathscr{F} \otimes \mathscr{F})$ carried by the diagonal by

$$
\tilde{\mu}(A \times B)=\mu(A \cap B) \quad(A, B \in \mathscr{F})
$$

or, equivalently,

$$
\tilde{\mu}(C)=\mu[\omega \in \Omega:(\omega, \omega) \in C] \quad(C \in \mathscr{F} \otimes \mathscr{F}) .
$$

(2.4) Definition. A state $\mu$ is called a Gibbs state for the interaction $U$ and the activity $z \geqq 0$ if $\mu\left(\Omega_{r}\right)=1$ and for any $\Lambda \in \mathscr{L}_{\sigma}$

$$
d \tilde{\mu} / d(\pi \otimes \mu) \mid \mathscr{F}_{\Lambda} \otimes \mathscr{F}_{S \backslash \Lambda}(\zeta, \omega)=f_{\Lambda}^{z}(\zeta \mid \omega) \quad \text { for } \quad \pi \otimes \mu-\text { a.a. }(\zeta, \omega)
$$

or, equivalently, for any $A \in \mathscr{F}$

$$
\mu\left[A \mid \mathscr{F}_{S \backslash A}\right]=\int_{A} \pi(d \zeta) f_{\Lambda}^{z}(\zeta \mid .) \quad \mu \text {-a.s. }
$$

Of course, this definition of Gibbs states is nothing else than that by the socalled DLR-equations.

We denote by $\mathfrak{5}(z)$ the convex set of all Gibbs states for $z$ and $U$ and by ex $\mathfrak{5}(z)$ the set of all extremal points of $\mathfrak{5}(z)$.

(2.6) Remark. $\mathfrak{G}(0)=\left\{\varepsilon_{0}\right\}$ where $\varepsilon_{0}$ is the unit mass on the empty configuration 0 . If $U$ is of the form $(\mathrm{PP})_{\theta},(\mathrm{DP})_{\theta},(\mathrm{CP})_{\theta}$, or $(\mathrm{WR})_{\theta}$ then $(\mathfrak{5}(z) \neq \emptyset$ for any $z>0$.

Proof. The first statement is trivial. For the existence in the cases $(\mathrm{CP})_{\theta}$ and $(\mathrm{DP})_{\theta}$ see $[3,14,12]$. The cases $(\mathrm{WR})_{\theta}$ and $(\mathrm{PP})_{\theta}$ can be handled by the methods in $[12]$. 
(2.7) Theorem. If $\mathfrak{5}(z) \neq \emptyset$ then ex $\mathfrak{5}(z) \neq \emptyset . \mu \in \mathfrak{G}(z)$ is extremal in $\mathfrak{G}(z)$ if and only if $\mu(A)=0$ or 1 for any $A \in \widetilde{F}_{\infty}$. Any $\mu \in \mathfrak{G}(z)$ has a unique representation

$$
\mu=\int_{\operatorname{ex} \tilde{\mathfrak{E}(z)}} v P^{\mu}(d v)
$$

by a probability measure $P^{\mu}$ on ex $\mathfrak{G}(z)$.

Proof. Let $\left(5^{\prime}(z)\right.$ be the set of all states $\mu$ with property (2.5). Since $\Omega$ is a Polish space and (2.5) defines a system of probability kernels $\Pi_{\Lambda}$ from $\left(\Omega, \mathscr{F}_{S \backslash A}\right)$ to $\left(\Omega, \mathscr{F}_{\Lambda}\right), \Lambda \in \mathscr{L}_{\sigma}$, which is consistent according to (2.2), we can apply the MartinDynkin boundary construction in [5] leading to the results stated in the theorem with $\mathfrak{5}(z)$ replaced by $\mathfrak{b}^{\prime}(z)$. Thus it is enough to show that ex $\mathfrak{5}(z)=\operatorname{ex} \mathfrak{b}^{\prime}(z) \cap \Re$ where $\mathfrak{R}$ is the set of all states carried by $\Omega_{r}$. But this is obvious.

If $V \subset \Delta \in \mathscr{L}$ let

$$
f_{V \Delta}^{z}(\zeta \mid \omega)=\int \pi(d \alpha) f_{\Delta}^{z}\left(\zeta_{V} \alpha_{\Delta \backslash V} \mid \omega\right)
$$

be the probability density with respect to $\pi$ of the restriction to $\mathscr{F}_{V}$ of the Gibbs distribution in $\Delta$ with boundary condition $\omega$.

(2.9) Corollary. If $\mu \in \operatorname{ex~}\left(\mathfrak{5}(z)\right.$ then for any sequence $\Delta \uparrow S$ in $\mathscr{L}_{\sigma}$ and any $V \in \mathscr{L}_{\sigma}$ the following is true: For $\mu-$ a.a. $\omega \in \Omega$ the sequence $f_{V \Delta}^{z}(. \mid \omega)$ converges $\pi-a . s$. and in $L^{1}(\pi)$-norm to $d \mu / d \pi \mid \mathscr{F}_{V}$.

In other words: If $\Delta \uparrow S$ then for $\mu$-a.a. $\omega$ the Gibbs distribution in $\Delta$ with boundary condition $\omega$ converges to $\mu$ in the sense of the total variation norm on any $\mathscr{F}_{V}, V \in \mathscr{L}$.

Proof. Observe first that for any $\mu \in \mathfrak{G}(z)$

$$
d \tilde{\mu} / d(\pi \otimes \mu) \mid \mathscr{F}_{V} \otimes \mathscr{F}_{S \backslash \Delta}(\zeta, \omega)=f_{V \Delta}^{z}(\zeta \mid \omega) \text { a.s. }
$$

Thus the martingale convergence theorem asserts that if $\Delta \uparrow S$ then $f_{V \Delta}^{z}(. \mid$.) converges a.s. and in $L^{1}$-norm with respect to $\pi \otimes \mu$ to a function $f_{V}(. \mid$.) being measurable with respect to $\mathscr{F}_{V, \infty}=\bigcap_{\Delta \in \mathscr{L}} \mathscr{F}_{V} \otimes \mathscr{F}_{S \backslash \Delta}$.

Now let $\mu \in \operatorname{ex} \mathfrak{5}(z)$. Then

$$
\mathscr{F}_{V, \infty}=\mathscr{F}_{V} \otimes\{\Omega, \emptyset\} \pi \otimes \mu-\text { a.s. }
$$

Indeed, trivially we have ' $\supset$ '. Conversely, if $A \in \mathscr{F}_{V, \infty}$ consider the cuts $A_{\zeta}=$ $\{\omega \in \Omega:(\zeta, \omega) \in A\}$. By Fubini's theorem, $A_{\zeta} \in \mathscr{F}_{\infty}$, and the function $\zeta \rightarrow \mu\left(A_{\zeta}\right)$ is $\mathscr{F}_{V}$-measurable and takes only the values 0 or 1 according to (2.7). Thus again by Fubini's theorem it is seen that the set

$$
B=\left\{(\zeta, \omega): \mu\left(A_{\zeta}\right)=1\right\} \in \mathscr{F}_{V} \otimes\{\Omega, \emptyset\}
$$

$\pi \otimes \mu$-almost surely coincides with $A$.

Now we conclude from (2.10) that there is a $\mathscr{F}_{V}$-measurable function $f_{V}($. such that $f_{V}(\zeta \mid \omega)=f_{V}(\zeta)$ for $\pi \otimes \mu-$ a.a. $(\zeta, \omega)$, and for any $A \in \mathscr{F}_{V}$ we have

$$
\int_{A} \pi(d \zeta) f_{V}(\zeta)=\lim _{\Delta \uparrow S} \int_{A \times \Omega}(\pi \otimes \mu)(d \zeta, d \omega) f_{V \Delta}^{z}(\zeta \mid \omega)=\tilde{\mu}(A \times \Omega)=\mu(A)
$$

Hence $f_{V}=d \mu / d \pi \mid \mathscr{F}_{V}$. 
Finally, it follows from Fubini's theorem that for $\mu-$ a.a. $\omega \pi\left[f_{V \Delta}^{z}(. \mid \omega) \rightarrow f_{V}\right]=1$, and, since all these functions of $\zeta$ are probability densities, the a.s. convergence implies norm convergence, see the proof of Korollar 20.5 in [1], e.g.

Let us conclude this section with a remark concerning the shift invariant case. Let $\sigma=\lambda$ and $U(\omega)=U\left(\theta_{x} \omega\right)$ for all $\omega \in \Omega$ and $x \in S$. Then the densities $f_{\Lambda}^{z}(. \mid$.) are also shift invariant, and we can conclude from [5] that Theorem (2.7) remains valid if $\mathfrak{G}(z)$ is replaced by the set $\mathfrak{G}_{\theta}(z)$ of all translationally invariant Gibbs states and $\mathscr{F}_{\infty}$ by the $\sigma$-field of invariant sets. Thus the extreme points of $\boldsymbol{6}_{\theta}(z)$ are ergodic and limits of averaged Gibbs distributions in bounded volumes.

\section{A Differential Characterization of Gibbs States}

It is known that states for lattice systems are Gibbsian as soon as their one-point conditional probabilities are given by the Gibbs distributions [2]. Here we prove a similar result for continuum Gibbs states.

Let $\mu$ be a state. $\mu$ is said to be a point process of first order if its intensity measure

$$
\varrho(D)=\int \mu(d \omega) \omega(D) \quad(D \in \mathscr{S})
$$

exists, i.e., $\varrho(D)<\infty$ if $D \in \mathscr{L}$. Suppose that $\mu$ is of first order. Then the Campbell measure $\mu_{C}$ of $\mu$ is the measure on $(S \times \Omega, \mathscr{S} \otimes \mathscr{F})$ defined by

$$
\mu_{C}(D \times A)=\int_{A} \mu(d \omega) \omega(D) \quad(D \in \mathscr{S}, A \in \mathscr{F}) .
$$

By desintegration of $\mu_{C}$ one obtains (see [8]) that for any $x \in S$ there is a probability measure $\mu_{x}$ on $(\Omega, \mathscr{F})$ such that

$$
\mu_{C}(D \times A)=\int_{D} \varrho(d x) \mu_{x}(A) \quad(D \in \mathscr{S}, A \in \mathscr{F})
$$

$\mu_{x}$ has to be imagined as the conditional probability with respect to $\mu$ under the condition that the site $x$ is occupied. The following properties hold (see [8]):

(3.1) For any $g \in L^{1}\left(\mu_{C}\right)$

$$
\int d \mu_{C} g=\int \mu(d \omega) \int \omega(d x) g(x, \omega)=\int \varrho(d x) \int \mu_{x}(d \omega) g(x, \omega) \text {. }
$$

(3.2) For $\varrho$ - a.a. $x \in S, \mu_{x}[\omega \in \Omega: x \in \omega]=1$.

In particular, these facts hold for $\pi$ and its intensity measure $\sigma$.

(3.3) Lemma. Let $V \in \mathscr{L}_{\sigma}$ and $g$ be $\mathscr{F}_{V}$-measurable such that $N(\Delta) g \in L^{1}(\pi)$ for any $\Delta \in \mathscr{L}$. Then for $\sigma-$ a.a. $x \in S$

$\int \pi_{x}(d \omega) g(\omega)=\int \pi(d \omega) g(\omega x)$.

Proof. This is a simple computation, see also [10].

For any $x \in S$ define the measure $\mu_{x}^{0}$ carried by $\{\omega \in \Omega: x \notin \omega\}$ via

$$
\int \mu_{x}^{0}(d \omega) g(\omega)=\int \mu_{x}(d \omega) g(\omega \backslash\{x\})
$$

where $g$ is any bounded $\mathscr{F}$-measurable function. From (3.3) it is seen that for $\sigma-$ a.a. $x$ we have $\pi_{x}^{0}=\pi$. Since Gibbs states are conceived as locally modified Poisson states, we can hope that Gibbs states can be identified by observing how the measures $\mu_{x}^{0}$ differ from $\mu$. Let $U$ and $\sigma$ be as in the preceding section. 
(3.5) Theorem. Let $\mu$ be a state of first order. Then $\mu \in \mathfrak{G}(z)$ if and only if

(a) $\mu\left(\Omega_{r}\right)=1$,

(b) $\varrho \ll \sigma$ with density $r($.$) ,$

(c) $\mu_{x}^{0} \ll \mu$ for $\varrho-a . a$. $x \in S$,

(d) For $\sigma \otimes \mu-a \cdot a .(x, \omega)$

$$
z e^{-U(x \mid \omega)}=\left\{\begin{array}{lll}
0 & \text { if } & r(x)=0 \\
r(x)\left(d \mu_{x}^{0} / d \mu\right)(\omega) & \text { if } & r(x)>0 .
\end{array}\right.
$$

Proof. "only if". (a) holds by definition. For (b) observe that for any $V \in \mathscr{L}_{\sigma} \mu \ll \pi$ on $\mathscr{F}_{V}$ with density $f_{V}^{\mu}=\int \mu(d \omega) f_{V}^{z}(. \mid \omega)$. Thus we see from (3.1) that for all $D \in \mathscr{S}$, $D \subset V$,

$$
\varrho(D)=\int \pi(d \omega) f_{V}^{\mu}(\omega) \omega(D)=\int_{D} \sigma(d x) \int \pi_{x}(d \omega) f_{V}^{\mu}(\omega) .
$$

For the proof of (c) and (d) choose some $\Lambda \in \mathscr{L}_{\sigma}$ and $A$ and $B$ from a countable generator of $\widetilde{\mathscr{F}}_{\Lambda}$ and $\widetilde{\mathscr{F}}_{S \backslash \Lambda}$, respectively, and let $\Lambda \supset D \in \mathscr{S}$. Then

$$
\begin{aligned}
& \int_{D} \sigma(d x) r(x) \mu_{x}^{0}(A \cap B)=\int_{D} \varrho(d x) \int \mu_{x}(d \omega) 1_{A \cap B}(\omega \backslash\{x\}) \\
& =\int_{B} \mu(d \omega) \int_{D} \omega(d x) 1_{A}(\omega \backslash\{x\})=\int_{B} \mu(d \omega) \int \pi(d \zeta) f_{A}^{z}(\zeta \mid \omega) \int_{D} \zeta(d x) 1_{A}(\zeta \backslash\{x\}) \\
& =\int_{B} \mu(d \omega) \int_{D} \sigma(d x) \int \pi(d \zeta) f_{A}^{z}(\zeta x \mid \omega) 1_{A}(\zeta) \\
& =\int_{B} \mu(d \omega) \int_{A} \pi(d \zeta) f_{A}^{z}(\zeta \mid \omega) z \int_{D} \sigma(d x) \exp \left[-U\left(x \mid \zeta_{\Lambda} \omega_{S \backslash A}\right)\right] \\
& =\int_{B} \mu(d \omega) E_{\mu}\left[1_{A} z \int_{D} \sigma(d x) e^{\left.-U(x \mid \cdot) \mid \mathscr{F}_{S \backslash A}\right](\omega)}\right. \\
& =\int_{D} \sigma(d x) \int_{A \cap B} \mu(d \omega) z e^{-U(x \mid \omega)}
\end{aligned}
$$

"if". Fix some $\Lambda \in \mathscr{L}_{\sigma}$ and let $g_{\Lambda}(0 \mid \omega)=e^{\sigma(\Lambda)} \mu\left[\Omega_{\Lambda, 0} \mid \mathscr{F}_{S \backslash \Lambda}\right](\omega)$. We show that

$$
g_{\Lambda}(\zeta \mid \omega)=\left\{\begin{array}{l}
g_{\Lambda}(0 \mid \omega) z^{\zeta(\Lambda)} \exp \left[-U\left(\zeta_{\Lambda} \omega_{\partial \Lambda}\right)+U\left(\omega_{\partial \Lambda}\right)\right] \text { if } \quad \omega_{S \backslash \Lambda} \in \Omega_{r} \\
1 \quad \text { otherwise }
\end{array}\right.
$$

is a version of $d \tilde{\mu} / d(\pi \otimes \mu) \mid \mathscr{F}_{\Lambda} \otimes \mathscr{F}_{S \backslash \Lambda}(\zeta, \omega)$. To this end it is enough to show by induction on $N$ that for any $\mathscr{F}$-measurable bounded function $h$ carried by $\Omega_{\Lambda, N}$ the equality

$$
\int \mu(d \omega) h(\omega)=\int \mu(d \omega) \int \pi(d \zeta) g_{\Lambda}(\zeta \mid \omega) h\left(\zeta_{\Lambda} \omega_{S \backslash \Lambda}\right)
$$

holds. For $N=0$ this is true by definition. If $N>0$ it suffices to consider functions of the form $h=1_{A} 1_{B}$ where $B \in \mathscr{F}_{S \backslash A}$ and

$$
A=\bigcap_{j=1}^{k} \Omega_{\Lambda_{j}, N_{j}}
$$

for some measurable partition $\Lambda_{1} \cup \ldots \cup \Lambda_{k}=\Lambda$ and integers $N_{j} \geqq 0$ such that $\sum_{j} N_{j}=N$. Say, $N_{1}>0$. Let

$$
A_{1}=\Omega_{\Lambda_{1}, N_{1}-1} \cap \bigcap_{j=2}^{k} \Omega_{\Lambda_{j}, N_{j}} .
$$


Then

$$
\begin{aligned}
& \left.\int_{B} \mu(d \omega) \int_{A} \pi(d \zeta) g_{A}(\zeta \mid \omega)=\int_{B} \mu(d \omega) \frac{1}{N_{1}} \int_{\Lambda_{1}} \sigma(d x) \int_{A_{1}} \pi(d \zeta) g_{A}(\zeta \mid \omega) z e^{-U\left(x \mid \zeta_{A} \omega_{\delta} A\right.}\right) \\
& =\int_{\Lambda_{1}} \varrho(d x) \frac{1}{N_{1}} \int_{B} \mu(d \omega) 1_{A_{1}}(\omega) d \mu_{x}^{0} / d \mu(\omega) \\
& =\int_{\Lambda_{1}} \varrho(d x) \frac{1}{N_{1}} \int_{B} \mu_{x}(d \omega) 1_{A_{1}}(\omega \backslash\{x\})=\int_{B} \mu(d \omega) 1_{A}(\omega) \omega\left(\Lambda_{1}\right) / N_{1}=\mu(A \cap B) .
\end{aligned}
$$

If $\mu$ is a shift invariant point process of first order then $\mu_{x}=\theta_{x} \mu_{0}$ for $\varrho-$ a.a. $x \in S$, see [8], and $\mu_{0}$ is called the Palm measure of $\mu$. Furthermore, $\varrho=c \lambda$ for some $c \geqq 0$. Thus $\mu \in \mathfrak{b}_{\theta}(z)$ if and only if either $c=z=0$ or $c>0, \mu\left(\Omega_{r}\right)=1, \mu_{0}^{0} \ll \mu$, and $d \mu_{0}^{0} / d \mu=\frac{z}{c} e^{-U(0 \mid .)}$.

\section{Canonical Gibbs States}

The canonical Gibbs states are defined by a very similar procedure as the Gibbs states, but now the grand canonical Gibbs distributions are replaced by the canonical ones. Let $\sigma$ and $U$ be as in Section 2.

If $\Lambda \in \mathscr{L}_{\sigma}$ the canonical Gibbs distribution in $\Lambda$ with boundary condition $\omega \in \Omega$ and particle number $N \geqq 0$ is the probability measure on $\left(\Omega, \mathscr{F}_{A}\right)$ whose RadonNikodym density with respect to $\pi \mid \mathscr{F}_{A}$ is

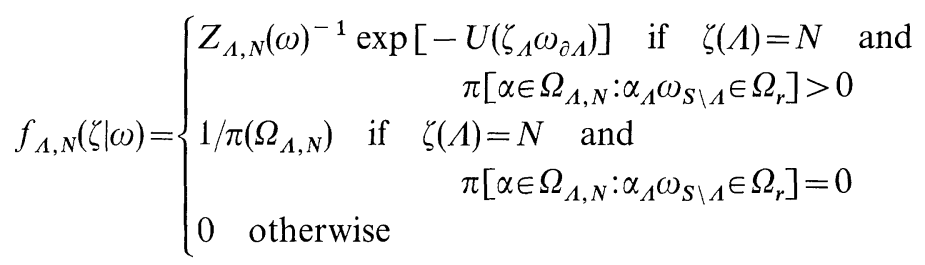

where the canonical partition function is given by

$$
\begin{aligned}
e^{\sigma(\Lambda)} Z_{\Lambda, N}(\omega) & =e^{\sigma(\Lambda)} \int_{\Omega_{\Lambda, N}} \pi(d \zeta) \exp \left[-U\left(\zeta_{\Lambda} \omega_{\partial \Lambda}\right)\right] \\
& =\frac{1}{N !} \int_{\Lambda^{N}} \sigma\left(d x_{1}\right) \ldots \sigma\left(d x_{N}\right) \exp \left[-U\left(x_{1} \ldots x_{N} \omega_{\partial \Lambda}\right)\right] .
\end{aligned}
$$

The canonical Gibbs densities satisfy the following consistency properties.

(4.2) Remark. (a) Whenever $\Lambda \subset \Delta \in \mathscr{L}, \sigma(\Lambda)>0, \omega \in \Omega$, and $N \geqq 0$ then for $\pi-$ a.a. $\zeta \in \Omega$

$$
f_{\Delta, N}(\zeta \mid \omega)=f_{A, \zeta(A)}\left(\zeta \mid \zeta_{\Delta} \omega_{S \backslash \Delta}\right) \int_{\Omega_{\Lambda, \zeta(A)}} \pi(d \alpha) f_{\Delta, N}\left(\alpha_{\Lambda} \zeta_{S \backslash \Lambda} \mid \omega\right) .
$$

(b) For any $\Lambda \in \mathscr{L}_{\sigma}, \omega \in \Omega$, and $z \geqq 0$

$$
f_{\Lambda}^{z}(\zeta \mid \omega)=f_{\Lambda, \zeta(\Lambda)}(\zeta \mid \omega) \int_{\Omega_{\Lambda, \zeta(\Lambda)}} \pi(d \alpha) f_{\Lambda}^{z}(\alpha \mid \omega) .
$$


Proof. (b) is easy. In order to prove (a) it suffices to consider the case $\zeta(\Delta)=N$. If $\pi\left[\alpha \in \Omega_{\Delta, N}: \alpha_{\Delta} \omega_{S \backslash \Delta} \in \Omega_{r}\right]=0$ then by Fubini's theorem for $\pi-$ a.a. $\zeta \in \Omega_{\Delta, N}$ we have $\pi\left[\beta \in \Omega_{\Lambda, \zeta(\Lambda)}: \beta_{\Lambda} \zeta_{\Delta \backslash \Lambda} \omega_{S \backslash A} \in \Omega_{r}\right]=0$ so that the assertion reduces to

$$
\pi\left(\Omega_{\Lambda, \zeta(\Lambda)}\right)^{-1} \pi\left(\Omega_{\Lambda, \zeta(\Lambda)}\right) / \pi\left(\Omega_{\Delta, N}\right)=\pi\left(\Omega_{\Delta, N}\right)^{-1} .
$$

Now let $\pi\left[\alpha \in \Omega_{\Delta, N}: \alpha_{\Delta} \omega_{S \backslash \Delta} \in \Omega_{r}\right]>0$ and fix some $n \leqq N$ and $\zeta^{0} \in \Omega_{\Delta \backslash \Lambda, N-n}^{0}$. If $\pi\left[\beta \in \Omega_{\Lambda, n}: \beta_{\Lambda} \zeta^{0} \omega_{S \backslash \Delta} \in \Omega_{r}\right]=0$ then the integral on the right hand side vanishes, and so does the left hand side for $\pi-$ a.a. $\zeta$ such that $\zeta_{\Delta \backslash \Lambda}=\zeta^{0}$. In the remaining case all terms are defined by the potentials, and the consistency follows from (U3).

Note that for any $\Lambda \in \mathscr{L}_{\sigma}$ the function

$$
(\zeta, \omega) \rightarrow f_{\Lambda, \omega(\Lambda)}(\zeta \mid \omega)
$$

is measurable with respect to $\mathscr{F}_{\Lambda} \otimes \mathscr{G}_{\Lambda}$.

(4.3) Definition. A state $\mu$ on $(\Omega, \mathscr{F})$ is called a canonical Gibbs state for the interaction $U$ if $\mu\left(\Omega_{r}\right)=1$ and for all $\Lambda \in \mathscr{L}_{\sigma}$

$d \tilde{\mu} / d(\pi \otimes \mu) \mid \mathscr{F}_{\Lambda} \otimes \mathscr{G}_{\Lambda}(\zeta, \omega)=f_{\Lambda, \omega(\Lambda)}(\zeta \mid \omega)$ a.s.

The convex set of all canonical Gibbs states with fixed interaction $U$ is denoted by $C$. Since $\mathscr{G}_{A}$ is generated by the sets of the form $\Omega_{\Lambda, N} \cap B$ where $N \geqq 0$ and $B \in \mathscr{F}_{S \backslash \Lambda}$, a state $\mu$ belongs to $\mathfrak{C}$ if and only if $\mu\left(\Omega_{r}\right)=1$ and for all $\Lambda \in \mathscr{L}_{\sigma}, N \geqq 0$, $B \in \mathscr{F}_{S \backslash \Lambda}$, and $A \in \mathscr{F}_{\Lambda}$

$$
\int_{A} \pi(d \zeta) \int_{B \cap \Omega_{\Lambda, N}} \mu(d \omega) f_{\Lambda, N}(\zeta \mid \omega)=\mu\left(A \cap B \cap \Omega_{\Lambda, N}\right) .
$$

In particular, $\mu \ll \pi$ on $\mathscr{F}_{\Lambda}$ with density

$$
f_{\Lambda}^{\mu}(\zeta)=\int \mu(d \omega) f_{\Lambda, \omega(\Lambda)}(\zeta \mid \omega)
$$

Since $\mathbb{b}(0)=\left\{\varepsilon_{0}\right\}$, the following proposition yields as a by-product that $\mathfrak{C} \neq \emptyset$, moreover, that ex $\mathbb{C} \neq \emptyset$.

(4.6) Proposition. For all $z \geqq 0, \mu \in \mathbb{5}(z)$ if and only if $\mu \in \mathbb{C}$ and for all $\Lambda \in \mathscr{L}_{\sigma}$, $N \geqq 0$, and $\mu-$ a.a. $\omega$

$$
(N+1) \mu\left[\Omega_{\Lambda, N+1} \mid \mathscr{F}_{S \backslash \Lambda}\right](\omega)=\int_{\Omega_{\Lambda, N}} \mu\left[d \zeta \mid \mathscr{F}_{S \backslash \Lambda}\right](\omega) \int_{\Lambda} \sigma(d x) z e^{-U\left(x \mid \zeta_{\Lambda} \omega_{\delta \Lambda}\right.} .
$$

Proof. First, let $\mu \in\left(\mathfrak{b}(z)\right.$ and $\Lambda \in \mathscr{L}_{\sigma}$. Then for any $A \in \mathscr{F}_{\Lambda}, B \in \mathscr{F}_{S \backslash \Lambda}$, and $N \geqq 0$ we obtain from (4.2) (b)

$$
\begin{aligned}
& \int_{A} \pi(d \zeta) \int_{B \cap \Omega_{\Lambda, N}} \mu(d \omega) f_{\Lambda, N}(\zeta \mid \omega) \\
& =\int_{A \cap \Omega_{\Lambda, N}} \pi(d \zeta) \int_{B} \mu(d \omega) f_{\Lambda, N}(\zeta \mid \omega) \int_{\Omega_{\Lambda, N}} \pi(d \alpha) f_{\Lambda}^{z}(\alpha \mid \omega) \\
& =\tilde{\mu}\left(\left(A \cap \Omega_{\Lambda, N}\right) \times B\right)=\mu\left(A \cap B \cap \Omega_{\Lambda, N}\right) .
\end{aligned}
$$

Hence $\mu \in \mathbb{C}$. The second property is trivial if $z=0$ and easily verified if $z>0$. 
Assume now that $\mu \in \mathfrak{C}$ has the stated property. In order to show that $\mu \in \mathbb{6}(z)$ it suffices to prove that if $z>0$ then for any $\Lambda \in \mathscr{L}_{\sigma}, N \geqq 0$, and $\pi \otimes \mu-$ a.a. $(\zeta, \omega) \in$ $\Omega_{\Lambda, N} \times \Omega$

$$
e^{-U\left(\omega_{\delta A}\right)} f_{\Lambda}^{\mu}(\zeta \mid \omega)=f_{\Lambda}^{\mu}(0 \mid \omega) z^{N} e^{-U\left(\zeta_{\Lambda} \omega_{\delta A}\right)}
$$

where

$$
\begin{aligned}
f_{\Lambda}^{\mu}(\zeta \mid \omega) & =d \tilde{\mu} / d(\pi \otimes \mu) \mid \mathscr{F}_{\Lambda} \otimes \mathscr{F}_{S \backslash \Lambda}(\zeta, \omega) \\
& =\mu\left[\Omega_{\Lambda, \zeta(\Lambda)} \mid \mathscr{F}_{S \backslash \Lambda}\right](\omega) f_{\Lambda, \zeta(\Lambda)}(\zeta \mid \omega) .
\end{aligned}
$$

This is done by induction on $N . N=0$ holds by definition. For the step $N \rightarrow N+1$ observe that a.s. on $\Omega_{A, N+1} \times \Omega_{r}$ the left hand side equals

$$
\begin{aligned}
& f_{\Lambda, N+1}(\zeta \mid \omega) \frac{z}{N+1} \int_{\Omega_{\Lambda, N}} \pi(d \alpha) e^{-U\left(\omega_{\delta A}\right)} f_{\Lambda}^{\mu}(\alpha \mid \omega) \int_{\Lambda} \sigma(d x) e^{-U\left(x \mid \alpha_{\Lambda} \omega_{\delta \Lambda}\right)} \\
& =f_{\Lambda}^{\mu}(0 \mid \omega) z^{N+1} f_{\Lambda, N+1}(\zeta \mid \omega) Z_{\Lambda, N+1}(\omega) \\
& =f_{\Lambda}^{\mu}(0 \mid \omega) z^{N+1} e^{-U\left(\zeta_{\Lambda} \omega_{\delta \Lambda}\right)} .
\end{aligned}
$$

The last equality holds a.s. since if $\omega_{S \backslash \Lambda} \in \Omega_{r}$ then $Z_{A, N+1}(\omega)=0$ if and only if $U\left(\zeta_{\Lambda} \omega_{\partial \Lambda}\right)=\infty$ for $\pi-$ a.a. $\zeta \in \Omega_{\Lambda, N+1}$ if and only if $\pi\left[\zeta \in \Omega_{\Lambda, N+1}: \zeta_{\Lambda} \omega_{S \backslash \Lambda} \in \Omega_{r}\right]=0$.

(4.7) Theorem. $\mu \in \mathbb{C}$ is extremal in $\mathbb{C}$ if and only if $\mu(A)=0$ or 1 for any $A \in \mathscr{G}_{\infty}$. Any $\mu \in \mathbb{C}$ has a unique representation

$$
\mu=\int_{\operatorname{ex} \mathbb{C}} v P^{\mu}(d v)
$$

by a probability measure $P^{\mu}$ on ex $\mathbb{C}$.

Proof. This statement fits into the setting of [5] just as Theorem (2.7). The probability kernels $P_{\Lambda}(\omega, A)$ from $\left(\Omega, \mathscr{G}_{\Lambda}\right)$ to $(\Omega, \mathscr{F})$ are defined by

$$
P_{\Lambda}(\omega, A \cap B)=1_{B}(\omega) \int_{A} \pi(d \zeta) f_{A, \omega(\Lambda)}(\zeta \mid \omega) \quad\left(A \in \mathscr{F}_{\Lambda}, B \in \mathscr{F}_{S \backslash A}\right)
$$

and if $\Lambda \subset \Delta \in \mathscr{L}, \sigma(\Lambda)>0$, it is easy to deduce from (4.2) (a) the consistency property $P_{\Delta} P_{A}=P_{\Delta}$ or, more explicitly,

$$
P_{\Delta}(\omega, A \cap B \cap C)=\int P_{\Delta}(\omega, d \zeta) P_{\Lambda}(\zeta, A \cap B \cap C)
$$

whenever $\omega \in \Omega, A \in \mathscr{F}_{\Lambda}, B \in \mathscr{F}_{\Delta \backslash \Lambda}, C \in \mathscr{F}_{S \backslash \Delta}$. Thus (4.7) follows from [5] just as (2.7).

If $V \subset \Delta \in \mathscr{L}_{\sigma}$ let

$$
f_{V \Delta, \omega(\Delta)}(\zeta \mid \omega)=\int \pi(d \alpha) f_{\Delta, \omega(\Delta)}\left(\zeta_{V} \alpha_{S \backslash V} \mid \omega\right)
$$

be the probability density with respect to $\pi \mid \mathscr{F}_{V}$ of the restriction to $\mathscr{F}_{V}$ of the canonical Gibbs distribution in $\Delta$ with boundary condition $\omega$ and particle number $\omega(\Delta)$.

(4.9) Corollary. If $\mu \in \operatorname{ex} \mathbb{C}$ then for any sequence $\Delta \uparrow S$ in $\mathscr{L}_{\sigma}$ and any $V \in \mathscr{L}_{\sigma}$ the following statement holds: For $\mu-$ a.a. $\omega$ the sequence $f_{V \Delta, \omega(\Delta)}(. \mid \omega)$ converges to $f_{V}^{\mu}=d \mu / d \pi \mid \mathscr{F}_{V} \pi$-a.s. and in $L^{1}(\pi)$-norm. 
In other words: Any extremal canonical Gibbs state is the limit of canonical Gibbs distributions in $\Delta$ with pure boundary condition $\omega$ and particle number $\omega(\Delta)$ in the sense of the total variation norm on any $\mathscr{F}_{V}, V \in \mathscr{L} . \omega$ can be chosen from a set of measure one.

Proof. Obviously,

$$
f_{V \Delta, \omega(\Delta)}(\zeta \mid \omega)=d \tilde{\mu} / d(\pi \otimes \mu) \mid \mathscr{F}_{V} \otimes \mathscr{G}_{\Delta}(\zeta, \omega)
$$

so that the same arguments apply as in the proof of (2.9).

In the shift invariant case one obtains from [5] that the extremal points of the set $\mathfrak{C}_{\theta}$ of all shift invariant canonical Gibbs states are just the ergodic states in $\mathfrak{C}_{\theta}$, and these are limits of averaged canonical Gibbs distributions in bounded volumes.

\section{The Activity Indicating Function}

Let $\sigma$ be an atomless Radon measure on $(S, \mathscr{S})$ and $U$ an interaction energy satisfying the following condition.

(A) $\sigma$ is infinite $e^{5}$ and one of the following assumptions holds.

(A1) Either $U \equiv 0$ or $U$ is of type (WR). In the latter case, $\sigma \leqq c \lambda$ for some $c>0$.

(A2) $U$ has the form (PP) or (DP), and $\sigma$ has property (S) and satisfies the inequality $\sigma \leqq c \lambda$ for some $c>0$.

(A3) $U$ is given by (CP), and there is a nice subset $S_{0}$ of $S$ (e.g., $S_{0}=S$ or a halfspace) and constants $c, c^{\prime}>0$ such that $c^{\prime} \lambda \leqq \sigma \leqq c \lambda$ on $S_{0}$ and $\sigma\left(S \backslash S_{0}\right)=0^{6}$. Furthermore, we introduce a certain temperedness condition for states ${ }^{7}$. Let

$$
t(\omega)=\lim _{\Delta \uparrow S} \sup \omega(\Delta) / \sigma(\Delta)
$$

be the (upper) particle density of $\omega, t_{0}=\infty$, and for $r>0 t_{r}=\sup \left\{t(\omega): \omega \in \Omega_{r}\right.$, $\left.\omega\left(S \backslash S_{0}\right)=0\right\}$. Define

$$
T=\left\{\omega \in \Omega_{r}: t(\omega)<t_{r}\right\}
$$

where $r$ is chosen according to property (U2). We call a state tempered and write $\mu \in \mathfrak{I}$ if $\mu(T)=1$.

We are going to identify any $\mu \in \mathfrak{C} \cap \mathfrak{I}$ as a mixture of Gibbs states. Obviously, we have

(5.1) Remark. $\operatorname{ex}(\mathfrak{C} \cap \mathfrak{I})=(\operatorname{ex} \mathfrak{C}) \cap \mathfrak{I}$.

If $U \equiv 0$ then $\mathfrak{C} \subset \mathfrak{I}$, see [11]. For any $\zeta, \omega \in \Omega$ and $V \in \mathscr{L}$ let

$$
z_{V}(\zeta \mid \omega)=\left\{\begin{aligned}
\lim \sup _{\Delta \perp S} \frac{Z_{\Delta \backslash V, \omega(\Delta)-\zeta(V)-1}\left(\zeta_{V} \omega_{S \backslash V}\right)}{Z_{\Delta \backslash V, \omega(\Delta)-\zeta(V)}\left(\zeta_{V}\left(\omega_{S \backslash V}\right)\right.} & \\
& \text { if } \zeta_{V} \in \Omega_{r} \text { and eventually } \omega_{S \backslash \Delta} \in \Omega_{r}, \omega(\Delta)>\zeta(V) \text { and the } \\
& \text { denominator }>0
\end{aligned}\right.
$$

See [7] for a discussion why this is necessary.

This implies condition (S).

A similar condition has been used in [14]. 
and

$$
z(\omega)=\limsup _{\Delta \perp S} z_{V}(\omega \mid \omega) \in[0, \infty]
$$

Since $U$ has finite range, the function $z($.$) is measurable with respect to the \sigma$-field generated by $\mathscr{F}_{\infty}$ and the set $\Omega_{r}$. We shall see that for any tempered canonical Gibbs state the activity distribution is given by the distribution of $z($.$) . In view$ of the Legendre relation between the canonical and the grand canonical pressure the following remark illustrates why $z($.$) is a good candidate for the activity.$

(5.4) Remark. Let $\sigma=\lambda$ and $U \equiv 0$ or of type $(\mathrm{DP})_{\theta}$ or $(\mathrm{CP})_{\theta}$. Then for any $\omega \in T$ we have

$$
-\log z(\omega)=\frac{\partial}{\partial \varrho} g(t(\omega), U)
$$

where $g(\varrho, U)$ is the specific Helmholtz free energy per volume. In particular, $z() \mid$.$T is shift invariant.$

Proof. A theorem by Dobrushin and Minlos [4] asserts that in the cases $(\mathrm{DP})_{\theta}$ and $(\mathrm{CP})_{\theta}$ for any $V \in \mathscr{L}$

$$
\log \frac{Z_{\Delta \backslash V, \omega(\Delta \backslash V)}(\omega)}{Z_{\Delta \backslash V, \omega(\Delta \backslash V)-1}(\omega)} \rightarrow \frac{\partial}{\partial \varrho} g(\varrho, U)
$$

whenever $\Delta$ runs through a subsequence of $\overline{\mathscr{L}}$ such that $\omega(\Delta) / \lambda(\Delta) \rightarrow \varrho$. Actually, they do not include a boundary condition, but to do so is no problem in the case $(\mathrm{CP})_{\theta}$, and for the case (DP) $)_{\theta} \mathrm{cf}$. Lemma (6.6) below. If $U \equiv 0$, this result is an easy computation. Now the remark follows from the concavity of the function $g(., U)$.

Sections 6 and 7 are devoted to the proof of the following key result.

(5.5) Proposition. Let $\mu \in \operatorname{ex} \mathfrak{C} \cap \mathfrak{I}$. Then there is an activity $z \in[0, \infty[$ such that $\mu[z()=z]=$.1 and $\mu \in \operatorname{ex} \mathfrak{5}(z) \cap \mathfrak{I}$.

Now the same argument as in [7] shows

(5.6) Theorem. ex $\mathfrak{C} \cap \mathfrak{I}=\bigcup_{0 \leqq z \leqq \infty} \operatorname{ex}(\mathfrak{H}(z) \cap \mathfrak{T}$.

If $U \equiv 0$ we can forget about the temperedness, and $\mathbb{C}$ is the set of all states with the property that if you know that $N$ particles are in some $\Lambda \in \mathscr{L}_{\sigma}$ then these particles are independently distributed according to $\sigma(. \mid \Lambda)$. Furthermore, $\mathfrak{b}(z)$ then consists just of the Poisson point process with intensity measure $z \sigma$. Thus Theorem (5.6) yields a characterization of the mixed Poisson point processes for $\sigma$ by the property defining $\mathfrak{C}$. This is a continuum analogue of de Finetti's theorem.

A convenient special formulation of Theorem (5.6) is given in

(5.7) Remark. If $\mathfrak{G}(z) \cap \mathfrak{I}=\left\{v^{z}\right\}$ for all $z \geqq 0$ then any $\mu \in \mathfrak{C} \cap \mathfrak{I}$ has a representation

$$
\mu=\int_{[0, \infty[} v^{z} P^{\mu}(d z)
$$

where $P^{\mu}$ is the distribution of $z($.$) under \mu$.

As in [7] now one derives the following characterization of tempered Gibbs states in the class of tempered canonical Gibbs states. 
(5.8) Theorem. For any $z \geqq 0, \mu \in \mathfrak{G}(z) \cap \mathfrak{I}$ if and only if $\mu \in \mathfrak{C} \cap \mathfrak{I}$ and $\mu[z()=$. $z]=1$.

Theorem (5.8) expresses in a certain sense the equivalence of ensembles. This becomes more clear in part (a) of the following corollary concerning the shift invariant case.

(5.9) Corollary.(a) In the situation of remark (5.4) the following statement holds for any $z \geqq 0: \mu \in \mathbb{5}(z) \cap \mathfrak{T}$ if and only if $\mu \in \mathfrak{C}$ and $\frac{\partial}{\partial \varrho} g(t(), U)=.-\log z \mu-$ a.s.

(b) Whenever $z() \mid$.$T is shift invariant, in particular, if the statement of (5.4) is$ valid, we have

$$
\operatorname{ex} \mathfrak{C}_{\theta} \cap \mathfrak{T}=\bigcup_{0 \leqq z \leqq \infty} \operatorname{ex} \mathfrak{G}_{\theta}(z) \cap \mathfrak{T}
$$

Proof. (a) follows from (5.8) and (5.4) since $\frac{\partial}{\partial \varrho} g(\varrho, U)>-\infty$ only if $\varrho<t_{r}$. (b) follows from (5.8) because any $\mu \in \operatorname{ex} \mathfrak{C}_{\theta}$ is ergodic.

It is an interesting consequence of Theorem (5.6) that tempered canonical Gibbs states are Gibbs states in a certain sense.

(5.10) Proposition. A state $\mu \in \mathfrak{T}$ belongs to $\mathfrak{C}$ if and only if for all $\Lambda \in \mathscr{L}_{\sigma}$

$$
d \tilde{\mu} / d(\pi \otimes \mu) \mid \mathscr{F}_{\Lambda} \otimes \mathscr{F}_{S \backslash \Lambda}(\zeta, \omega)=f_{\Lambda}^{z}{ }^{(\omega)}(\zeta \mid \omega) \text { a.s. }
$$

Proof. Denote by $\mathfrak{D}$ the convex set of all states $\mu$ with this property. Then $\mathfrak{D} \subset \mathbb{C}$ by the same argument as in the first part of the proof of (4.6). On the other hand, it follows from (5.5) that ex $\mathfrak{C} \cap \mathfrak{I} \subset \mathfrak{D}$, hence by (4.7) $\mathfrak{C} \cap \mathfrak{I} \subset \mathfrak{D}$.

As in [7] we now obtain

(5.11) Theorem. For any $\mu \in \mathfrak{C} \cap \mathfrak{I}, \mathscr{G}_{\infty}=\mathscr{F}_{\infty} \mu-$ a.s.

\section{Some Estimates}

In this section we prepare the proof of Proposition (5.5) by some estimates most of which are modifications of results of Dobrushin and Minlos $[4]^{8}$. We shall assume throughout that condition $(\mathrm{A})$ is satisfied.

We consider the non-normalized partition functions

$$
\begin{aligned}
Q_{\Delta, N}(\omega) & =N ! e^{\sigma(\Delta)} Z_{\Delta, N}(\omega) \\
& =\int_{\Delta^{N}} \sigma\left(d x_{1}\right) \ldots \sigma\left(d x_{N}\right) \exp \left[-U\left(x_{1} \ldots x_{N} \omega_{\partial \Delta}\right)\right]
\end{aligned}
$$

where $\Delta \in \mathscr{L}, \omega_{S \backslash \Delta} \in \Omega_{r}$, and $N \geqq 0$.

8 Our estimates here probably are not the best possible. An extension to a larger class of interactions automatically extends the range of validity of Theorem (5.6). 
(6.2) Lemma. For all $t<t_{r}$ there is a constant $K(t)>0$ such that for any sequence $\left(N_{k}\right)_{k \geqq 1}$ with the property $\limsup _{k \rightarrow \infty} N_{k} / \sigma(\Delta(k)) \leqq t$, any sequence $\left(\omega_{k}\right)_{k \geqq 1}$ in $\Omega_{r}$, all $V \in \overline{\mathscr{L}}$, and sufficiently large $\Delta_{k}=\Delta(k) \backslash V$

$$
Q_{\Delta_{k}, N_{k}+1}\left(\omega_{k}\right) / Q_{\Delta_{k}, N_{k}}\left(\omega_{k}\right) \geqq K(t) \sigma\left(\Delta_{k}\right) .
$$

Proof. Just as in [4] we have only to show that if $k$ is large, $\zeta \in \Omega_{\Delta_{k}, N_{k}}^{0}, \zeta \omega_{S \backslash \triangle_{k}} \in \Omega_{r}$, and $\zeta\left(S \backslash S_{0}\right)=0$

$$
\int_{\Delta_{k}} \sigma(d x) \exp \left[-U\left(x \mid \zeta \omega_{\partial \Delta_{k}}\right)\right] \geqq K(t) \sigma\left(\Delta_{k}\right)
$$

for some $K(t)>0$. In case (A1) this is trivial since then $U(x \mid) \leqq$.0 .

Consider now the case (A3). Choose some $\delta<t_{r}-t$. Write $\Delta$ instead of $\Delta_{k} \cap S_{0}$ and let

$$
\Delta(\zeta)=\{x \in \Delta:\|x-y\|>r \text { for all } y \in \zeta\}
$$

and $M=\max N(\Delta)\left(\Omega_{r}\right)-N_{k}$. Then there is some $\alpha \in \Omega_{\Delta, M}^{0} \cap \Omega_{r}$ such that

$$
\lambda(\Delta(\zeta)) \geqq \lambda\left(\bigcup_{x \in \alpha}\{y \in \Delta:\|y-x\| \leqq r\}\right) .
$$

Hence, if $\Delta$ is large enough,

$$
\sigma(\Delta(\zeta)) / \sigma(\Delta) \geqq c^{\prime} v_{r} \kappa M / \sigma(\Delta) \geqq c^{\prime} v_{r} \kappa \delta=4 D
$$

where $v_{r}=\lambda[y \in S:\|y\| \leqq r]$ and $\kappa$ is the minimal fraction of this volume within a large cube containing the origin. Now choose $a>r$ so small that $(t+\delta) c\left(v_{a}-v_{r}\right)<D$ and $k$ so large that $N_{k} / \sigma(\Delta)<t+\delta$ and $\sigma((\Delta \backslash \Delta([k-R])) \cup \partial V) \leqq D \sigma(\Delta)$. Then the set

$$
\Delta_{a}(\zeta)=\{x \in \Delta([k-R]) \backslash \bar{V}:\|x-y\| \geqq a \text { for all } y \in \zeta\}
$$

satisfies the inequality

(6.4) $\sigma\left(\Delta_{a}(\zeta)\right) \geqq 2 D \sigma(\Delta)$.

Furthermore, we have due to (P2), (P1), and (U3)

$$
\begin{aligned}
\int_{\Delta_{a}(\zeta)} \sigma(d x) \mid U\left(x \mid \zeta \omega_{S \backslash \Delta_{k}}\right) & \leqq \sum_{y \in \zeta} \int_{|| x-y \| \geqq a} \sigma(d x)|\Phi(x, y)| \\
& \leqq N_{k} K_{a} \leqq \sigma(\Delta)(t+\delta) K_{a}=E \sigma(\Delta) .
\end{aligned}
$$

Thus we obtain from (6.4) and Cebyshev's inequality

$$
\begin{aligned}
\sigma\left[x \in \Delta: U\left(x \mid \zeta \omega_{S \backslash \Delta_{k}}\right)\right. & \leqq E / D] \\
& \geqq \sigma\left(\Delta_{a}(\zeta)\right)-\sigma\left[x \in \Delta_{a}(\zeta): U\left(x \mid \zeta \omega_{S \backslash \Delta_{k}}\right)>E / D\right] \geqq D \sigma(\Delta)
\end{aligned}
$$

proving (6.3) with $K(t)=D \exp [-E / D]$.

In the case (A2) the same argument simplifies since we can choose $D=1 / 4$.

(6.5) Corollary. For any $\omega \in T$ and $\zeta \in \Omega$,

$$
\sup _{V \in \mathscr{\mathscr { L }}} z_{V}(\zeta \mid \omega)<\infty
$$


Proof. We can assume that $\zeta \in \Omega_{r}$ and $\omega(S)>\zeta(V)$ for any $V \in \overline{\mathscr{L}}$. Fix some $V$ and let $N_{k}=\max (0, \omega(\Delta(k))-\zeta(V)-1)$ and $\omega_{k}=\zeta_{V} \omega_{S \backslash \Delta(k)}$. Then

$$
\limsup _{k \rightarrow \infty} N_{k} / \sigma(\Delta(k))=t(\omega)<t_{r},
$$

and

$$
z_{V}(\zeta \mid \omega)=\limsup _{k \rightarrow \infty}\left(N_{k}+1\right) \frac{Q_{\Delta_{k}, N_{k}}\left(\omega_{k}\right)}{Q_{\Delta_{k}, N_{k}+1}\left(\omega_{k}\right)} \leqq t(\omega) / K(t(\omega)) .
$$

(6.6) Lemma. If $U$ is of type (DP) then for all $\omega \in T$ and $v>0$ there are constants $D<\infty, \delta<0$ such that for any cube $\Lambda$ with $\lambda(\Lambda)=v$, all $l \geqq 0$, and sufficiently large $\Delta \in \overline{\mathscr{L}}$

$$
\int_{\{N(\Lambda) \geqq l\}} \pi(d \zeta) f_{\Delta, \omega(\Delta)}(\zeta \mid \omega) \leqq D e^{-\delta l^{2}},
$$

Proof. Essentially this is Theorem 3 in [4]. We indicate some modifications of the proof.

1. Let $C$ be as in (P1) and $H=C 2^{R+1} 2^{d}(R+1)^{d}$. Determine some $\left.a \in\right] 0,1[$ such that $1 / a$ is an integer and

$$
\psi(r) r^{d} \geqq 8 H d^{d / 2} \quad \text { if } \quad r \leqq a d^{1 / 2} .
$$

Then if $x, y$ belong to a cube of vertex length $a$,

$$
\Phi(x, y) \geqq 8 H / v
$$

where $v=a^{d}$ is the volume of the cube.

2. Divide $S$ in cubic cells of vertex length $a$. We denote cells as well as their center by $\gamma$. Let $\Gamma$ be the set of cells in $\Delta$. If $x=\left(x_{1}, \ldots, x_{d}\right) \in S$ let $|x|=\max _{1 \leqq i \leqq d}\left|x_{i}\right|$. Define

$$
\Phi\left(\gamma, \gamma^{\prime}\right)=\inf _{x \in \gamma, x^{\prime} \in \gamma^{\prime}} \Phi_{-}\left(x, x^{\prime}\right) .
$$

Then since $\Phi$ has range $R$ we have for any cell $\gamma$

$$
\sum_{\gamma^{\prime}} \Phi\left(\gamma, \gamma^{\prime}\right) 2^{\left|\gamma^{\prime}-\gamma\right|} \geqq-H / v \text {. }
$$

3. Choose $\Lambda \in \Gamma$. Then $l_{0}=\sup _{\gamma} \omega(\gamma) 2^{-|\gamma-\Lambda|}<\infty$. Indeed, suppose that there is a sequence $\gamma_{i}$ such that $\left|\gamma_{i}\right| \uparrow \infty$ and $\omega\left(\gamma_{i}\right) 2^{-\left|\gamma_{i}\right|} \geqq 1$. Let $k_{i}$ be the smallest integer $\geqq\left(1+\left|\gamma_{i}\right|\right)$. Then

$$
\omega\left(\Delta\left(k_{i}\right)\right) / \sigma\left(\Delta\left(k_{i}\right)\right) \geqq \omega\left(\gamma_{i}\right) /\left(c \lambda\left(\Delta\left(k_{i}\right)\right)\right) \geqq \frac{1}{c} 2^{\left|\gamma_{i}\right|}\left(2 k_{i}\right)^{-d} \rightarrow \infty
$$

in contradiction to the assumption $t(\omega)<\infty$. and

4. Fix some $l \geqq l_{0}$. For any $G \subset \Gamma$ let $(G)=\bigcup_{\gamma \in G} \gamma, \mathcal{N}(G)=\left\{n \in \mathbb{Z}^{G}: n(\gamma) \geqq \mid 2^{|\gamma-\Lambda|}\right\}$

$$
A(G, n)=\left\{\zeta \in \Omega_{\Delta, \omega(\Delta)}^{0}: \zeta(\gamma)=n(\gamma) \quad \text { if } \quad \gamma \in G, \zeta(\gamma)<l 2^{|\gamma-\Lambda|} \quad \text { if } \quad \gamma \notin G\right\} .
$$


Then the event $\{N(\Lambda) \geqq l\}$ is the disjoint union of the events $A(G, n), A \in G \subset \Gamma$, $n \in \mathscr{N}(G)$.

5. In order to estimate

$$
\int_{A(G, n)} \pi(d \zeta) f_{\Delta, \omega(\Delta)}(\zeta \mid \omega)
$$

we write for any $\zeta \in \Omega_{\Delta, \omega(\Delta)}$ with $\zeta_{S \backslash \Delta}=\omega_{S \backslash \Delta}$

$$
U\left(\zeta_{\bar{\Delta}}\right) \geqq \sum_{\gamma \in G} U\left(\zeta_{\gamma}\right)+U\left(\zeta_{\bar{\Delta} \backslash(G)}\right)+\sum_{\gamma \in G, \gamma^{\prime} \subset \bar{\Delta}} \zeta(\gamma) \zeta\left(\gamma^{\prime}\right) \Phi\left(\gamma, \gamma^{\prime}\right) .
$$

The last sum has the lower bound

$$
-\frac{2 H}{v} \sum_{\gamma \in G} \zeta(\gamma)^{2} .
$$

This follows from 2. and 3. by similar estimates as in [4]. Combined with 1. and Lemma (6.2) we then obtain by the arguments in [4] for (6.7) an upper bound of the form

$$
\exp \left[-\delta \sum_{\gamma \in G} n(\gamma)^{2}\right]
$$

where $\delta>0$. This proves the Lemma via the same reasoning as in [4].

Now we show that extremal tempered canonical Gibbs states are of first order. It is clear that extremality is necessary. As an example take the canonical Gibbs state $\int_{0}^{\infty} \pi_{z} v(d z)$ for the potential $U \equiv 0$ where $v$ is a probability measure with infinite expectation and $\pi_{z}$ the Poisson state with intensity measure $z \sigma$.

(6.8) Proposition. Any $\mu \in \operatorname{ex} \mathfrak{C} \cap \mathfrak{I}$ is of first order, i.e., its intensity measure

$$
\varrho(\Lambda)=\int \mu(d \omega) \omega(\Lambda) \quad(\Lambda \in \mathscr{S})
$$

is finite if $\Lambda \in \mathscr{L}$.

Proof. The assertion is trivial in the case (A3). Consider now the cases (A1) and (A2) (PP) in which the potential is strongly stable. Then for any $\Lambda \in \overline{\mathscr{L}}$, sufficiently large $\Delta \in \overline{\mathscr{L}}$, and $\omega \in T$ we have with the abbreviation $N=\omega(\Delta)$

$$
\begin{aligned}
& \int \pi(d \zeta) \zeta(\Lambda) f_{\Delta, \omega(\Delta)}(\zeta \mid \omega) \\
&=\left(e^{-\sigma(\Delta)} / N !\right) \int_{\Delta^{N}} \sigma\left(d x_{1}\right) \ldots \sigma\left(d x_{N}\right) \sum_{i=1}^{N} 1_{\Lambda}\left(x_{i}\right) f_{\Delta, N}\left(x_{1} \ldots x_{N} \mid \omega\right) \\
&=Q_{\Delta, N}(\omega)^{-1} N \int_{\Delta^{N-1}} \sigma\left(d x_{1}\right) \ldots \sigma\left(d x_{N-1}\right) \exp \left[-U\left(x_{1} \ldots x_{N-1} \omega_{\partial \Delta}\right)\right] \\
& \quad \cdot \int_{\Lambda} \sigma(d x) \exp \left[-U\left(x \mid x_{1} \ldots x_{N-1} \omega_{\partial \Delta}\right)\right] \\
& \leqq \sigma(\Lambda) e^{B} N Q_{\Delta, N-1}(\omega) / Q_{\Delta, N}(\omega) \\
& \leqq \sigma(\Lambda) e^{B} K(t(\omega))^{-1} \omega(\Delta) / \sigma(\Delta)
\end{aligned}
$$


the last inequality being a consequence of (6.2). In the case (A2) (DP) it follows from (6.6) that

$$
\int \pi(d \zeta) \zeta(\Lambda) f_{\Delta, \omega(\Delta)}(\zeta \mid \omega) \leqq D \sum_{l=1}^{\infty} e^{-\delta l^{2}}<\infty .
$$

Now we see from (4.9) and Fatou's lemma that for some $\omega \in T$

$$
\begin{aligned}
\int \mu(d \zeta) \zeta(\Lambda) & =\int \pi(d \zeta) \zeta(\Lambda) \lim _{\Delta \uparrow S} f_{\Delta, \omega(\Delta)}(\zeta \mid \omega) \\
& \leqq \liminf _{\Delta \unlhd S} \int \pi(d \zeta) \zeta(\Lambda) f_{\Delta, \omega(\Delta)}(\zeta \mid \omega)<\infty .
\end{aligned}
$$

\section{Investigation of Extremal Tempered Canonical Gibbs States}

Suppose that condition (A) holds, and fix some $\mu \in \operatorname{ex} \mathfrak{C} \cap \mathfrak{I}$. Consider its intensity measure $\varrho$ on $(S, \mathscr{S})$ which is a Radon measure due to (6.8). Furthermore, remind that for any $V \in \mathscr{L}_{\sigma} \mu \ll \pi$ on $\mathscr{F}_{V}$ with density (4.5). Then it is seen just as in (3.5) that $\varrho \ll \sigma$. We denote the density which is the first correlation function of $\mu$ by $r($.$) . In order to be complete and to simplify the reasoning below we show$

(7.1) Proposition. Either $\varrho=0$ or $\varrho \sim \sigma$.

Proof. Suppose that $\varrho(S)>0$. Then $\lim _{\Delta \unlhd S} \mu\left(\Omega \backslash \Omega_{\Delta, 0}\right)=\mu[\omega: \omega(S) \geqq 1]=2 a>0$. Choose $\Delta_{0}$ such that if $\Delta \supset \Delta_{0}$ then $\mu\left(\Omega \backslash \Omega_{\Delta, 0}\right) \geqq a$. Then in the case (A3) the temperedness of $\mu$ guarantees that there is some $\delta>0$ and $\Delta_{1} \supset \Delta_{0}$ such that $\mu[N(\Delta) /$ $\sigma(\Delta) \leqq\left(t_{r}-\delta\right)(1-\delta)$ for all $\left.\Delta_{1} \subset \Delta \in \overline{\mathscr{L}}\right] \geqq 1-a / 2$. Thus we can find arbitrarily large $\Delta \in \overline{\mathscr{L}}$ such that $\mu\left(A_{\Delta}\right) \geqq a / 2>0$, where

$$
A_{\Delta}=\left(\Omega \backslash \Omega_{\Delta, 0}\right) \cap\left\{N(\Delta) \leqq(1-\delta) \max N\left(\Delta \cap S_{0}\right)\left(\Omega_{r}\right)\right\} \in \mathscr{G}_{\Lambda} .
$$

Now for any $D \in \mathscr{S}, D \subset \Delta$ we have

$$
\mu[N(D)>0] \geqq \int_{A_{\Delta}} \mu(d \omega) \int_{\{N(D)>0\}} \pi(d \zeta) f_{\Delta, \omega(\Delta)}(\zeta \mid \omega) .
$$

But for sufficiently large $\Delta$ and $\omega \in A_{\Delta}$ the $\omega(\Delta)$ hard balls in $\Delta$ can move rather freely, that is, if $\sigma(D)>0$ at least one of them will be able to occupy $D$, i.e., the inner integral on the right is positive. Since $\mu\left(A_{\Delta}\right)>0$ this proves that $\varrho(D)>0$. In the cases (A1) and (A2) the integral on the right is trivially positive for all $\Delta \supset \Delta_{0}$.

If $\varrho=0$ then $\mu=\varepsilon_{0}$. Furthermore, $z()=.0 \varepsilon_{0}$-a.s. Thus Proposition (5.5) is true in this case. Hence we shall suppose now that $\mu \neq \varepsilon_{0}$. Then $0<r(x)<\infty$ for $\sigma-$ a.a. $x \in S$.

(7.2) Proposition. $\mu[\omega \in \Omega: \omega(S)=\infty]=1$.

Proof. Since $\{N(S)=\infty\} \in \mathscr{G}_{\infty}$ this event has measure 0 or 1 . Assume that $\mu[N(S)<\infty]=1$. Then there is some integer $N$ such that $\mu[N(S)=N]=1$ because these events are symmetric, too. $\mu \neq \varepsilon_{0}$ implies $N \geqq 1$. Fix some $V \in \mathscr{L}_{\sigma}$. Then obviously $\mu[N(V)>N]=0$. Fix some $\omega$ for which $\omega(S)=N$ and $\lim _{\Delta \perp S} f_{V \Delta, \omega(\Delta)}(. \mid \omega)=$ $f_{V}^{\mu} \pi$-a.s. In particular, $f_{V}(0)=\lim _{\Delta \pm S} f_{V \Delta, \omega(\Delta)}(0 \mid \omega)$. Thus for any $0 \leqq n<N$ and 
$\pi$-a.a. $\zeta \in \Omega_{V, N-n}$ we have

$$
\begin{aligned}
f_{V}^{\mu}(0) / f_{V}^{\mu}(\zeta) & =\lim _{\Delta \perp S} f_{V \Delta, N}(0 \mid 0) / f_{V \Delta, N}(\zeta \mid 0) \\
& =\frac{n !}{N !} \lim _{\Delta \perp S} \frac{(\Delta \backslash V)^{N}}{\int_{(\Delta \backslash V)^{n}} \sigma\left(d x_{1}\right) \ldots \sigma\left(d x_{N}\right) e^{-U\left(x_{1} \ldots x_{N}\right)}} \\
& \geqq \frac{n !}{N !} \inf _{\zeta(S)=N-n, \alpha(S)=n, \alpha \in \Omega_{r}} e^{U(\zeta \alpha)-U(\alpha)} \liminf _{\Delta \perp S} Q_{\Delta \backslash V, N}(0) / Q_{\Delta \backslash V, n}(0) .
\end{aligned}
$$

The infimum is positive, and according to (6.2) the lim inf is infinite. Hence $f_{V}^{\mu}=0$ a.s. on $\{N(V)>0\}$ for any $V$ and thereby $\mu=\varepsilon_{0}$.

In order to show that $\mu \in \mathfrak{G}(z)$ for some $z>0$ we intend to apply Theorem (3.5). Thus we have to identify the function $z(.) e^{-U(x \mid .)} / r(x)$ as the Radon-Nikodym derivative $d \mu_{x}^{0} / d \mu$.

Fix some $\Lambda \in \mathscr{L}_{\sigma} \cdot z_{V}(. \mid$.$) is defined by (5.2).$

(7.3) Lemma. Let $V \in \overline{\mathscr{L}}_{\sigma}, V \supset \bar{\Lambda}$.

(a) For $\sigma \otimes \pi \otimes \mu$-almost all $(x, \zeta, \omega) \in \Lambda \times \Omega \times \Omega$

$$
f_{V}^{\mu}(\zeta x)=z_{V}(\zeta \mid \omega) \exp \left[-U\left(x \mid \zeta_{V}\right)\right] \quad f_{V}^{\mu}(\zeta) .
$$

(b) For $\sigma \otimes \mu$-almost all $(x, \omega) \in \Lambda \times \Omega$

$$
f_{V}^{\mu}(\omega x) / f_{V}^{\mu}(\omega)=z_{V}(\omega \mid \omega) e^{-U(x \mid \omega)} .
$$

Proof. "(a)" Observe that due to (4.9), (6.5), and (7.2) the right-hand side for all $x \in \Lambda$ and $\pi \otimes \mu$-a.a. $(\zeta, \omega) \in \Omega \times T$ is the limit of

$$
\frac{Z_{\Delta \backslash V, \omega(\Delta)-\zeta(V)-1}\left(\zeta_{V} \omega_{S \backslash V}\right)}{Z_{\Delta \backslash V, \omega(\Delta)-\zeta(V)}\left(\zeta_{V} \omega_{S \backslash V}\right)} e^{-U\left(x \mid \zeta_{V}\right)} f_{V \Delta, \omega(\Delta)}(\zeta \mid \omega)
$$

if $\zeta_{V} \in \Omega_{r}$ and vanishes otherwise. But if $\omega \in T$ then for sufficiently large $\Delta$ the first case in (4.1) occurs, and if $\Delta \supset V \supset \bar{\Lambda}$ then there is no interaction between $\Lambda$ and $\Delta \backslash V$. Thus the expression above equals

$$
f_{V \Delta, \omega(\Delta)}(\zeta x \mid \omega)
$$

for large $\Delta$ and $x \notin \zeta$ which happens for $\pi$-a.a. $\zeta$. But this expression converges $\sigma \otimes \pi \otimes \mu$-almost surely to the lefthand side of (a). Indeed, from (3.3) and Fubini's theorem we deduce

$$
\begin{aligned}
& \int_{\Lambda} \sigma(d x) \pi \otimes \mu\left[(\zeta, \omega): f_{V \Delta, \omega(\Delta)}(\zeta x \mid \omega) \rightarrow f_{V}^{\mu}(\zeta x)\right] \\
& =\int_{\Lambda} \sigma(d x) \int \mu(d \omega) \pi_{x}\left[f_{V \Delta, \omega(\Delta)}(. \mid \omega) \rightarrow f_{V}^{\mu}\right] \\
& =\int \mu(d \omega) \int \pi(d \zeta) 1_{\left\{f_{V \Delta, \omega(\Delta)}(. \mid \omega) \rightarrow f_{V}^{\mu}\right\}}(\zeta) \zeta(\Lambda)=\sigma(\Lambda) .
\end{aligned}
$$

“(b)" Since $\tilde{\mu} \ll \pi \otimes \mu$ on $\mathscr{F}_{V} \otimes \mathscr{G}_{V}$ by definition of canonical Gibbs states, we may replace in (a) $\pi \otimes \mu$ by $\tilde{\mu}$. But due to (2.3) this is nothing else than (b). Note that $f_{V}^{\mu}>0 \mu-$ a.s. 
(7.4) Lemma. Let $V \in \overline{\mathscr{L}}_{\sigma}, V \supset \bar{\Lambda}$. Then for $\sigma-$ a.a. $x \in \Lambda \mu_{x}^{0} \ll \mu$ on $\mathscr{F}_{V}$ with density $f_{x, V}^{0}(\zeta)=r(x)^{-1} z_{V}(\zeta \mid \omega) \exp \left[-U\left(x \mid \zeta_{V}\right)\right]$

where $\omega \in T$ is a fixed configuration.

Proof. For all $D \in \mathscr{S}, D \subset V$, and all $A$ belonging to a countable generator of $\mathscr{F}_{V}$ we obtain from (3.1) and (3.3)

$$
\begin{aligned}
\int_{D} \sigma(d x) r(x) \mu_{x}^{0}(A) & =\int_{D} \varrho(d x) \int \mu_{x}(d \zeta) 1_{A}(\zeta \backslash\{x\}) \\
& =\int \pi(d \zeta) \int_{D} \zeta(d x) 1_{A}(\zeta \backslash\{x\}) f_{V}^{\mu}(\zeta)=\int_{D} \sigma(d x) \int_{A} \pi(d \zeta) f_{V}^{\mu}(\zeta x) .
\end{aligned}
$$

Thus for $\sigma-$ a.a. $x$ we have $\mu_{x}^{0} \ll \pi$ on $\mathscr{F}_{V}$ with density $f_{V}^{\mu}(\zeta x) / r(x)$. But $(6.5)$ and (7.3) (a) imply that $f_{V}^{\mu}(\zeta x)=0$ for $\pi$-a.a. $\zeta \in\left\{f_{V}^{\mu}=0\right\}$. This proves that $\mu_{x}^{0} \ll \mu$ on $\mathscr{F}_{V}$ with density $f_{V}^{\mu}(\zeta x) /\left(f_{V}^{\mu}(\zeta) r(x)\right)$. Now apply again (7.3) (a) and the fact that $\mu(T)=1$.

The proof of Proposition (5.5) is completed by (3.5), (7.1), and

(7.5) Proposition. For $\sigma-$ a.a. $x \in S, \mu_{x}^{0} \ll \mu$ with density

$$
d \mu_{x}^{0} / d \mu=r(x)^{-1} z e^{-U(x \mid \cdot)}
$$

where the constant $z>0$ is determined by the property $\mu[z()=z]=$.1 .

Proof. We have to extend the local absolute continuity established in (7.4) to a global one. A simple argument (see [9], Lemma 2.1) shows that a sufficient condition is

$$
\lim _{V \perp S} \sup _{x} d \mu_{x}^{0} / d \mu \mid \mathscr{F}_{V}<\infty \quad \mu_{x}^{0}-\text { a.s. }
$$

Thus we have to show that for any given $\Lambda \in \mathscr{L}_{\sigma}$ and $\sigma$-a.a. $x \in \Lambda$

$$
\limsup _{V \perp S} f_{x, V}^{0}<\infty \quad \mu_{x}^{0}-\text { a.s. }
$$

Note that for $\sigma-$ a.a. $x \in \Lambda \mu_{x}^{0}\left[\zeta \in \Omega: \zeta x \in \Omega_{r}\right]=1$ since

$$
\varrho(\Lambda)=\int_{\Omega_{r}} \mu(d \omega) \omega(\Lambda)=\int_{\Lambda} \varrho(d x) \int \mu_{x}^{0}(d \zeta) 1_{\Omega_{r}}(\zeta x) .
$$

Thus for $\mu_{x}^{0}-$ a.a. $\zeta$ the factor $\exp [-U(x \mid \zeta)]$ is well-defined and finite and equals $\exp \left[-U\left(x \mid \zeta_{V}\right)\right]$ if $V \supset \bar{\Lambda}$. Thus it follows from (6.5) that $\mu_{x}^{0} \ll \mu$ for $\sigma-$ a.a. $x \in S$.

Now recall (7.4) and (7.3) (b) and use the martingale convergence theorem in order to see that

$$
\begin{aligned}
\left(d \mu_{x}^{0} / d \mu\right)(\omega) & =\lim _{V \perp S} r(x)^{-1} z_{V}(\omega \mid \omega) e^{-U(x \mid \omega)} \\
& =r(x)^{-1} z(\omega) e^{-U(x \mid \omega)}
\end{aligned}
$$

for $\mu-$ a.a. $\omega \in \Omega$. But now (4.7) implies that $z()=.z \mu-$ a.s. for some $z \geqq 0$. But $z=0$ would imply that $\mu=\varepsilon_{0}$. 


\section{References}

1. Bauer,H.: Wahrscheinlichkeitstheorie und Grundzüge der Maßtheorie. Berlin: de Gruyter 1968

2. Dobrushin, R.L.: Theory Probability Appl. 13, 197-224 (1968)

3. Dobrushin, R. L.: Theor. math. Physics 4, 705-719 (1970)

4. Dobrushin, R.L., Minlos, R. A.: Theory Probability Appl. 12, 535-559 (1967)

5. Föllmer,H.: In: Seminaire de Probabilités IX, Lecture Notes in Mathematics 465, Berlin-Heidelberg-New York: Springer 1975

6. Georgii,H.O.: Z. Wahrscheinlichkeitstheorie verw. Gebiete 32, 277-300 (1975)

7. Georgii, H.O.: Z. Wahrscheinlichkeitstheorie verw. Gebiete 33, 331-341 (1976)

8. Krickeberg, K.: Fundamentos del analisis estadistico de procesos puntuales, Lecture Notes, Santiago de Chile 1973

9. Liggett, Th. M.: Trans. Amer. Math. Soc. 179, 433-453 (1973)

10. Nguyen,X.X., Zessin,H.: Punktprozesse mit Wechselwirkung, Thesis, Bielefeld 1975

11. Nguyen, X.X., Zessin,H.: Martin-Dynkin boundary of mixed Poisson processes and phase transition, preprint 1975

12. Preston, C. J.: Random fields, Lecture Notes, Oxford 1975

13. Ruelle, D.: Statistical Mechanics. New York-Amsterdam: Benjamin 1969

14. Ruelle, D.: Commun. math. Phys. 18, 127-159 (1970)

15. Shiga,T.: Some problems related to Gibbs states, canonical Gibbs states and Markovian time evolutions, preprint 1975

16. Widom, B., Rowlinson, J.S.: J. Chem. Phys. 52, 1670-1684 (1970)

Communicated by G. Gallavotti

Received December 13, 1975 
